\title{
IS THERE ANY ASSOCIATION OF GLUTATHIONE S-TRANSFERASE T1 (GSTT1) AND GLUTATHIONE S-TRANSFERASE M1 (GSTM1) GENE POLYMORPHISM WITH GASTRIC CANCERS?
}

\author{
Aptullah Haholu ${ }^{1}$, Ufuk Berber ${ }^{1}$, Bülent Karagöz ${ }^{2}$, Tolga Tuncel ${ }^{2}$, OĞuz Bilgi², \\ Dilaver Demirel ${ }^{1}$
}

${ }^{1}$ Pathology Department, Gulhane Military Medical Academy (GMMA) Haydarpasa Training Hospital, Istanbul, Turkey
${ }^{2}$ Oncology Department, Gulhane Military Medical Academy (GMMA) Haydarpasa Training Hospital, Istanbul, Turkey

\begin{abstract}
The glutathione S-transferases (GST) are enzymes catalyzing reactions including carcinogens. GSTT1 and GSTM1 genes are polymorphic in humans. The relations between polymorphism of some GST genes and cancer have been reported. In this study, we aimed to investigate the distribution of GSTT1 and GSTM1 polymorphisms in a group of gastric cancer patients. The study group consisted of 50 patients $(21 \mathrm{fe}-$ males, 29 males) with gastric adenocarcinoma from the archives of the pathology department of a training hospital. Fifty-seven healthy control subjects were included in the study as a control group. DNA was extracted from peripheral venous blood of control subjects and from the paraffin blocks of cases. Genotyping of GSTT1 and GSTM1 genes was performed with duplex polymerase chain reaction. No differences in the frequencies of GSTM1 or GSTT1 null genotypes were observed between patients and healthy subjects (GSTM1: $52 \%$ vs. $43.85 \%, \mathrm{OR}=1.27,95 \% \mathrm{CI}: 0.55$ 2.96; GSTT1: $38.46 \%$ vs. $28.07 \%$, OR $=0.72$, $95 \%$ CI: $0.25-1.96)$. Moreover, simultaneous carriage of both genotypes was almost identical in both groups. GSTM1 or GSTT1 null genotypes were not different in diffuse or intestinal type gastric cancer. Our data suggest that the GSTM1 and GSTT1 polymorphisms are not associated with gastric cancer in a small group of the Turkish population.
\end{abstract}

Key words: gastric cancer, genetic polymorphism, glutathione S-transferase.

\section{Introduction}

Gastric cancer (GC) is one of the most common cancer types and one of the leading causes of cancer-related deaths in the world. It is considered to be an important public health problem. Although the mechanism of gastric carcinogenesis is still not fully understood, some susceptibility genes have been described. When combined with environmental factors, these susceptibility genes including metabolic genes may be important in the development of cancer [1-5]. Metabolic genes produce some enzymatic and structural proteins that are responsible for metabolizing as well as detoxifying varying amounts of environmental carcinogens. Polymorphisms in these genes naturally may lead to alterations in their products, causing variety in their metabolizing and detoxifying ability. Insufficient elimination of carcinogens is believed to contribute to development of cancer and individual variation may cause individual susceptibility to different cancer types [6]. The glutathione S-transferases (GSTs) superfamily is one of the families of metabolic genes. The enzymatic protein products of these genes are involved in phase II conjugation reactions. They are known to catalyze reactions between glutathione and different types of toxic compounds [7]. Another role of these enzymes is believed to be the protection of DNA from oxidative damage [8, 9].

Many studies have been performed to detect the association between genetic polymorphisms of GSTs and cancer risk of various organs [6, 10-12]. It is reported that, in humans, there are eight distinct gene fam- 
ilies encoding the GSTs. They are $\alpha$ (GSTA), $\mu$ (GSTM), $\vartheta$ (GSTT), $\pi$ (GSTP), $\varsigma$ (GSTZ), $\sigma$ (GSTS), $\kappa($ GSTK) and $\omega$ (GSTO). Special attention has been focused on allelism in the $\mu$ (GSTM) and $\vartheta$ (GSTT) families [13]. The presence of GSTM1 on at least one allele or its absence in both alleles constitutes the polymorphism. Its deletion in both alleles is called GSTM1 null genotype. It is expected that individuals with GSTM1 null genotype will have no capacity to detoxify certain substrates. The insufficient metabolism and elimination of toxic substrate in GSTM1 null genotype may also cause DNA damage, suggesting its role in carcinogenesis [14]. The $\vartheta$ class (GSTT) enzyme is reported to catalyze the detoxification of ethylene oxide and methyl bromide and activates halogenated metabolites. GSTT1 is also polymorphic, with a null GSTT1 allele and at least one "active" GSTT1 allele [15, 16].

In the present study, we aimed to investigate the status and distribution of GSTT1 and GSTM1 polymorphism in GC and its histologic subtypes.

\section{Material and methods}

\section{Study group}

The present study consisted of 50 subjects ( $21 \mathrm{fe}$ males, 29 males) with pathologically confirmed primary GC who were recruited from the archives of the Pathology Department of Gulhane Military Medical Academy (GMMA) Haydarpasa Training Hospital in Istanbul, from October 2004 to June 2011. Sex and age frequency-matched controls were randomly selected from the healthy people. A total of 57 healthy individuals (24 females, 33 males) were included in the study as a control group.

This study was performed according to the Declaration of Helsinki and has been approved by the local

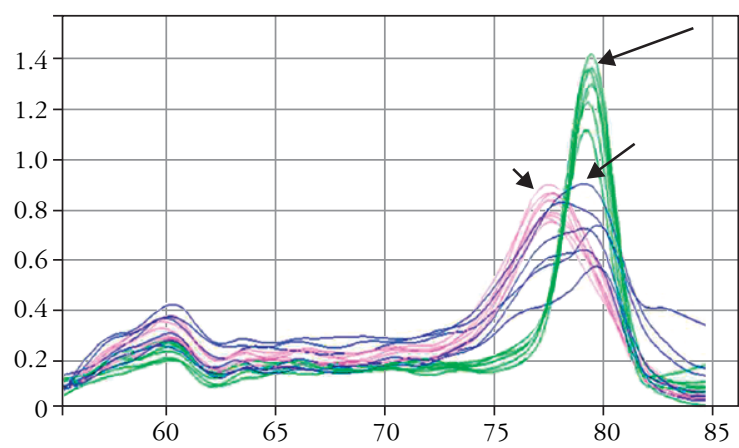

Fig. 1. Melting curves generated after multiplex PCR. The difference of $\sim 1.7^{\circ} \mathrm{C}$ in melting points enables the identification of GSTM1 and GSTT1 amplicons (long arrow: green lines correspond to GSTT1 amplicons; short arrow: pink lines correspond to GSTM1 amplicons; medium arrow: blue lines indicate presence of both genes; each line represents a case) ethics committee of the GATA Haydarpasa Training Hospital (No: 2012-11).

\section{Histopathologic examination}

Hematoxylin and eosin stained sections from formalin-fixed paraffin-embedded (FFPE) blocks sampled from gastrectomy materials were examined microscopically. Cases were divided into two groups, namely intestinal type and diffuse type according to the Lauren classification. In histopathological examination, while intestinal-type adenocarcinomas have glandular pattern, diffuse-type gastric adenocarcinomas are characterized by diffuse growth without clear gland formation. In many cases, the cells of diffuse type gastric adenocarcinoma are of the signet ring cell morphology $[17,18]$.

\section{DNA extraction and genotyping analysis}

Genomic DNA was isolated from FFPE blocks containing nontumoral stomach tissues and from cold blood of control subjects by using the QIAamp DNA FFPE tissue kit and the QIAamp DNA minikit (Qiagen, California, USA) respectively according to the manufacturer's instructions. To improve the efficacy of the PCR and to minimize the adverse effects of formalin on DNA content, we designed the primer sets to produce an amplicon of 75 bp length for GSTM1 and 70 bp length for GSTT1 genes flanking the fourth and sixth exons, respectively. Forward 5'ATGGTTTGCAGGAAACAAGG3' and reverse 5'CCTCCATAACACGTGAAGCA3' primers were used for amplification of the GSTM1 gene, and forward 5'TTCCTGGGTGAGCCAGTATC3' and reverse 5'ACTGCAGGGTCACATCCAA3' primers were used for the GSTT1 gene. The specificities of the primer pairs were analyzed using Primer-BLAST software (http://www.ncbi.nlm.nih.gov/tools/primer-blast/ index.cgi). PCR amplifications were carried out on Ro-

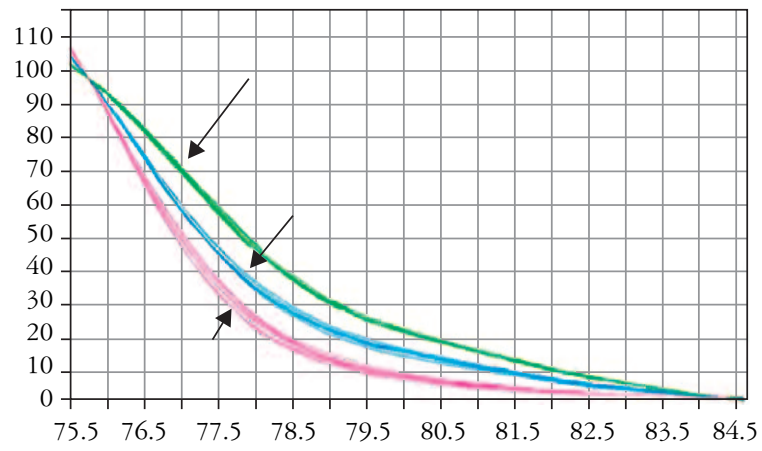

Fig. 2. HRMC analysis data obtained after multiplex PCR for screening null genotypes of GSTT1 and GSTM1 genes [green lines (long arrow) correspond to GSTT1 mplicons, pink lines (short arrow) correspond to GSTM1 amplicons, and blue lines (medium arrow) indicate presence of both genes] 

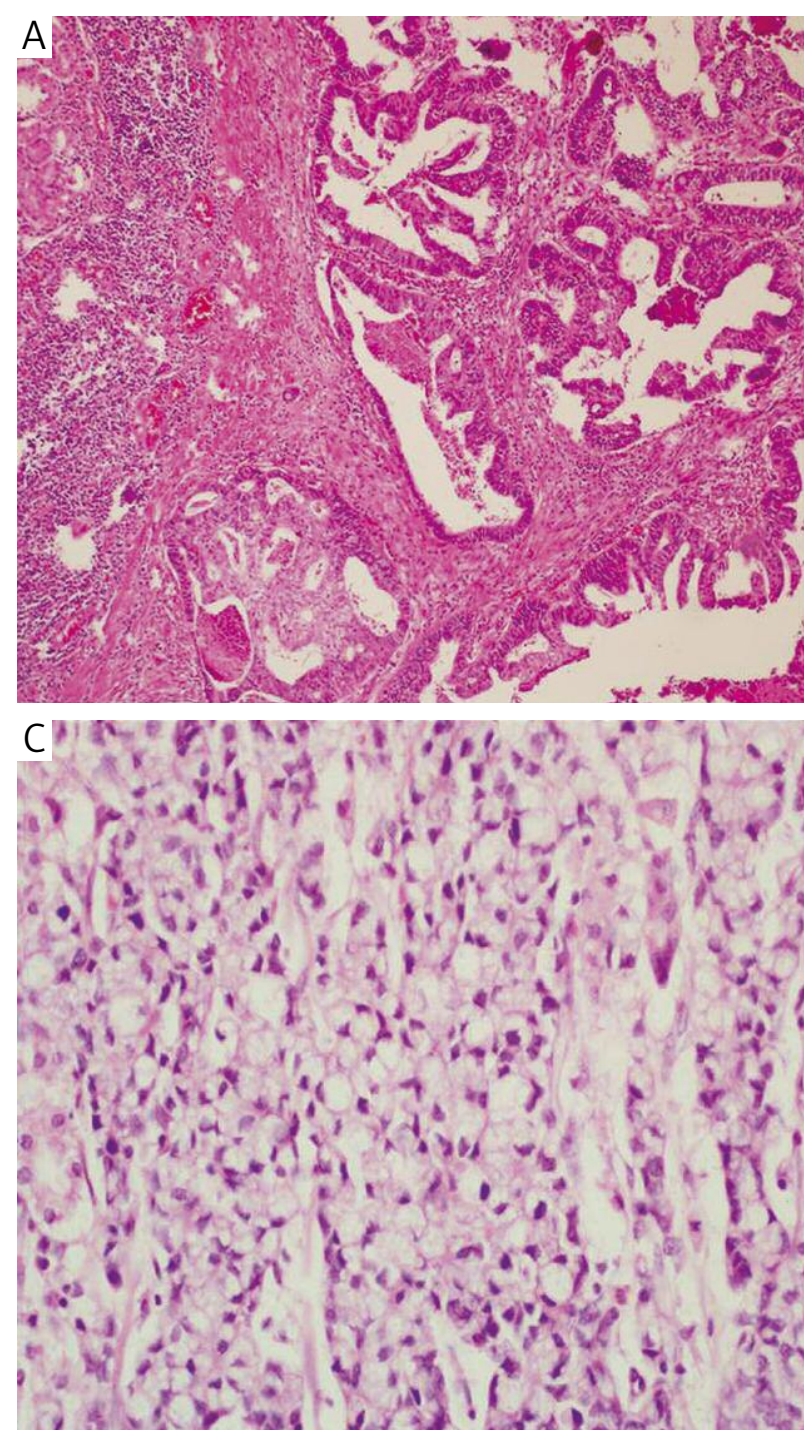

torGene Q 5-Plex in a final volume of $50 \mu \mathrm{l}$ containing 100 ng of genomic DNA.

We performed a multiplex polymerase chain reaction (PCR) amplification and high resolution melting curve (HRMC) analysis to screen null and wild type genotypes simultaneously in a single tube. The amplification protocol consisted of an activation step at $95^{\circ} \mathrm{C}$ for $15 \mathrm{~min}$ utes and 40 cycles with amplification steps at $94^{\circ} \mathrm{C}, 57^{\circ} \mathrm{C}$, and $72^{\circ} \mathrm{C}$ for $30 \mathrm{~s}$ each. Fluorescence acquisition was obtained at the annealing step. After amplification steps, both HRMC and melting curves were generated to evaluate PCR products. Melting curve data were acquired between $55^{\circ} \mathrm{C}$ and $85^{\circ} \mathrm{C}$, at a ramping rate of $0.6^{\circ} \mathrm{C} / \mathrm{s}$, and HRMC data were acquired between $74^{\circ} \mathrm{C}$ and $85^{\circ} \mathrm{C}$, at a ramping rate of $0.05^{\circ} \mathrm{C} / \mathrm{s}$. Normalized and subtracted $\mathrm{df} / \mathrm{dt}$ melting curves were visually compared to identify different melting patterns (Figs. 1, 2).

\section{Statistical analysis}

The relationship of the GSTM1 and GSTT1 genotypes with the risk of GC was assessed by means of odds

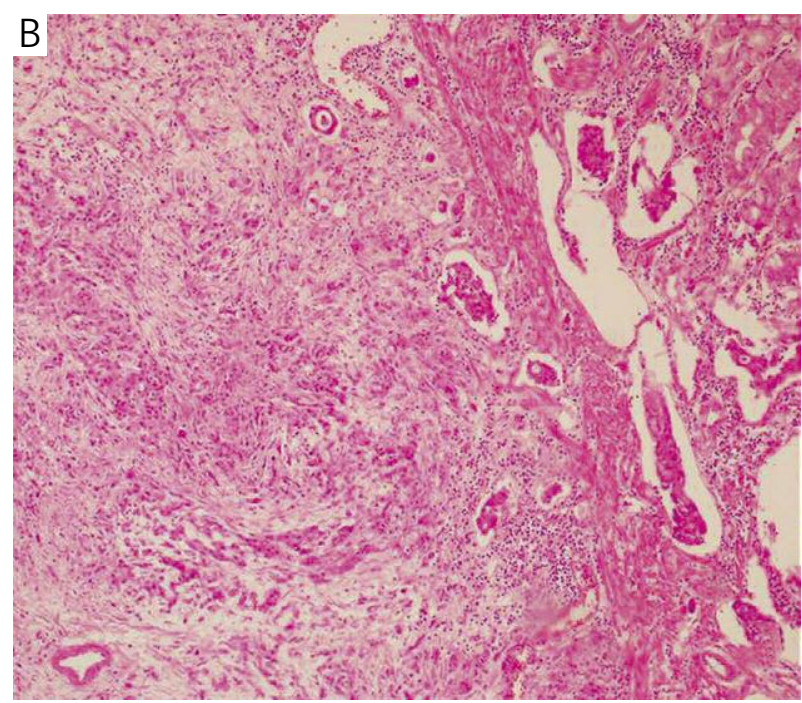

Fig. 3. Microscopic view of intestinal A) and diffuse B) type gastric adenocarcinomas A. Intestinal type adenocarcinoma is composed of atypical glandular structures (HE, 200×). B) Solid areas and single cell infiltration of tumor cells in diffuse type adenocarcinoma (HE, 100×). C) Some cases of diffuse type also showed signet ring cells $(\mathrm{HE}, 100 \times)$

ratios (OR) and 95\% confidence intervals (CI). GSTM1 and GSTT1 genotypes were classified as either null (homozygous deletion) or present. Statistical analyses were performed using the SPSS software. The univariate analyses to identify variables were investigated using Fisher's exact test. For multivariate analysis with adjustment for possible confounders, including sex and age, logistic regression analysis was used. $\mathrm{P}<0.05$ was considered statistically significant.

\section{Results}

Fifty GC patients were included in the study. Histopathologic examination revealed 25 diffuse type and 25 intestinal type. Some cases also had a mucinous component and signet ring cells (Fig. 1A-C). The mean age of the study group and the controls was $62.35 \pm 13.61$ years and $66.35 \pm 12.71$ years respectively.

According to the PCR results, out of 50 total cases, $24(48.0 \%)$ were GSTM1 positive and $26(52.0 \%)$ 
Table I. GSTM1 and GSTT1 genotypes in gastric cancer patients and healthy subjects

\begin{tabular}{lccccccc}
\hline & \multicolumn{2}{c}{ PATIENTS } & \multicolumn{5}{c}{ CONTROLS } \\
\hline & $\mathbf{N}$ & $(\%)$ & $\mathbf{N}$ & $(\%)$ & OR & CI (95\%) & P \\
\hline GSTM1 present & 24 & $(48.0)$ & 32 & $(56.1)$ & 1.27 & $(055-2.96)$ & 0.58 \\
\hline GSTM1 null & 26 & $(52.0)$ & 25 & $(43.9)$ & & & \\
\hline GSTT1 present & 40 & $(80.0)$ & 41 & $(71.9)$ & 0.72 & $(0.27-1.96)$ & 0.52 \\
\hline GSTT1 null & 10 & $(20.0)$ & 16 & $(28.1)$ & & & \\
\hline $\begin{array}{l}\text { Both GSTM1 } \\
\text { and GSTT1 null }\end{array}$ & 3 & $(6.0)$ & 0 & $(0)$ & 0.10 & & \\
\hline
\end{tabular}

Table II. GSTM1 and GSTT1 genotyping in diffuse and intestinal gastric cancer patients

\begin{tabular}{lccccc}
\hline CONTROL & DIFFUSE TYPE & INTESTINAL TYPE & OR & CI (95\%) & P* $^{*}$ \\
\hline GSTM1 null (\%) & 43.9 & 52.0 & 1.12 & $(0.40-3.11)$ & 0.82 \\
& & 52.0 & 1.89 & $(0.59-6.11)$ & 0.28 \\
\hline GSTT1 null (\%) & 28.1 & 16.0 & 0.50 & $(0.14-1.88)$ & 0.31 \\
& & 24.0 & 1.24 & $(0.33-4.67)$ & 0.76 \\
\hline Both GSTM1 & 0 & 2 & 1 & & \\
and GSTT1 null (cases) & & & & & \\
\hline
\end{tabular}

*Logistic regression test

were GSTM1 null. In the control group, GSTM1 positivity was $56.1 \%$ (32 of 57) and GSTM1 null was $43.9 \%$ ( 25 of 57 ) (GSTM1 null: OR $=1.27,95 \% \mathrm{CI}$ : $0.55-2.96 ; \mathrm{p}=0.58$ ). As for the GSTT1 study, 40 of 50 total cases $(80.0 \%)$ were positive, while the remaining 10 cases $(20.0 \%)$ were null. In the control group, GSTT1 positivity was $71.9 \%$ (41 of 57) and the frequency of GSTT1 null was $28.1 \%$ (16 of 57) (GSTT1 null: $\mathrm{OR}=0.72,95 \% \mathrm{CI}: 0.27-1.96 ; \mathrm{p}=0.52$ ). Three cases of GC patients were null in both GSTM1 and GSTT1 genotype, but none of the control subjects $(\mathrm{p}=0.10)$. GSTM1 and GSTT1 genotyping is summarized in Table I.

When intestinal type GC was compared to diffuse type, GSTM1 and GSTT1 null percentage was similar (GSTM1 null: $52 \%$ vs. 52\%; $\mathrm{p}=1.0$; GSTT1 null: $24 \%$ vs. $16 \% ; \mathrm{p}=0.72$; both GSTM1 and GSTT1 null: one case vs. two cases respectively). When GC patients were considered separately, either diffuse or intestinal type, GSTM1 and GSTT1 genotyping was not different from healthy subjects (Table II).

\section{Discussion}

As in many tumors, the exact mechanisms in human gastric tumorigenesis are still unknown. It is accepted that GC may be caused by a complex interaction of environmental and genetic factors [19-21]. Some epidemiological studies have suggested a relationship between genetic predisposition and GC tumorigenesis. Many studies have been carried out to understand the genetic background of gastric carcinogenesis.
Some of them are studies about the GST superfamily. Alterations or absence of GST enzyme activity in individuals result in insufficient elimination of DNA damaging compounds, which might lead to increased risk of somatic mutation causing tumorigenesis [14].

GSTM1 and GSTT1 genes, which are members of the GST superfamily, are believed to be involved in carcinogenesis [7]. However, the results of studies on the relationship between GSTM1 and GSTT1 genotyping and GC are controversial. Chen et al. found that GSTT1 null genotype was associated with a statistically significant increased risk of GC. They also showed that the frequency of GSTT1 null genotype was distinctly different between Asians and Caucasians [15]. The differences between ethnic groups concerning the association of GSTT1 genotypes and the risk of GC were also reported in other previous studies [22-24].

Several investigators have reported that GSTM1 null individuals are more susceptible to several cancers including skin cancer, lymphoma and leukemia, esophageal cancer, prostate cancer, colorectal cancer, and GC [24]. It is reported that GSTM1 gene polymorphism may be associated with GC risk among Asians [25]. In another study it was suggested that the GSTM1 null genotype is a low-penetrant risk factor for GC development in Asians [2].

We aimed to investigate the distribution of GSTT1 and GSTM1 polymorphisms in GC patients in this study. The polymorphism can be studied in blood or tissue sample. Derived from pathology archives, tissue blocks are available for genetic studies. In our study, we used archival tissue samples of gastrectomy mate- 
rials of cancer patients and blood of control subjects. Since we designed short amplicons for PCR, 75 bp length for GSTM1 and 70 bp length for GSTT1, the tissue fixation and paraffin blocking process is not expected to damage or prevent amplification of this size of DNA. DNA isolation was successful in samples from all subjects. PCR amplification of the target in positive cases was also successful. It supports the idea that archival tissue blocks are a valuable source for molecular studies, especially when the patient DNA from blood is not always accessible [26].

As previously stated, some ethnical differences may affect the gene polymorphism status and its relationship with certain types of cancer. Tamer et al. studied GSTM1, GSTT1 and GSTP1 gene polymorphisms and their association with smoking in a group of 70 patients from the Turkish population [27]. Their results suggested involvement of GSTM1 in GC; however, they suggest no association of GSTT1 and GSTP1 with GC. Their study was carried out in blood samples from both patients and control subjects.

To our knowledge, this is the first study of GSTT1 and GSTM1 polymorphisms comprising paraffin tissue blocks and blood samples together in a Turkish population. The subtypes of GCs, namely diffuse type and intestinal type, and their relationship with GSTM1 and GSTT1, were also investigated separately. The results suggest that the GSTM1 and GSTT1 genotypes are not associated with GC in the group studied. Although three cases of GC patients were null in both GSTM1 and GSTT1 genotype but none of the control subjects, this difference is not statistically significant. When histologic subtypes (diffuse or intestinal type) of GC were considered separately, GSTM1 and GSTT1 genotyping was not different from healthy subjects. Since the prognosis of diffuse gastric carcinoma is poorer than the intestinal one, we expected to see some differences in genetic polymorphism of studied genes. Still, these differences may be investigated by studies comprising more patients and with a different genetic target.

In conclusion, our data suggest that the GSTM1 and GSTT1 genotypes are not associated with GC in a small group of the Turkish population. When considering diffuse or intestinal type GC separately, GSTM1 and GSTT1 genotyping was also not different from healthy subjects. Several limitations, such as small numbers of patient and control groups, may be attributed to the study. On the other hand, outcome of patients, survival or other etiological and clinical data have not been included in the study. However, considering the genetic polymorphism and cancer relation of studied genes, the results may be valued as a contribution to the literature. Future studies that investigate GST genes in more patients and include other etiologic and clinical data may be useful to support the status of cancer and genetic polymorphism in the Turkish population.
The authors declare no conflict of interest.

\section{References}

1. Brenner H, Rothenbacher D, Arndt V. Epidemiology of stomach cancer. Methods Mol Biol 2009; 472: 467-477.

2. Qiu LX, Wang K, Lv FF, et al. GSTM1 null allele is a risk factor for gastric cancer development in Asians. Cytokine 2011; 55: $122-125$.

3. Nowara E, Boratyn-Nowicka A, Polakiewicz-Gilowska A, et al. Chemotherapy for gastric cancer patients - time for personalization in medicine? Contemp Oncol (Pozn) 2012; 16: 86-89.

4. Eljaszewicz A, Jankowski M, Gackowska L, et al. Gastric cancer increase the percentage of intermediate (CD14 ++ CD16+) and nonclassical (CD14+CD16+) monocytes. Centr Eur J Immunol 2012; 37: 355-361.

5. Brzozowa M, Mielańczyk L, Michalski M, et al. Role of Notch signaling pathway in gastric cancer pathogenesis. Contemp Oncol (Pozn) 2013; 17: 1-5.

6. López-Cima MF, Alvarez-Avellón SM, Pascual T, et al. Genetic polymorphisms in CYP1A1, GSTM1, GSTP1 and GSTT1 metabolic genes and risk of lung cancer in Asturias. BMC Cancer 2012; 12: 433.

7. Masoudi M, Saadat I, Omidvari S, Saadat M. Genetic polymorphisms of GSTO2, GSTM1, and GSTT1 and risk of gastric cancer. Mol Biol Rep 2009; 36: 781-784.

8. Ntais C, Polycarpou A, Ioannidis JP. Association of GSTM1, GSTT1, and GSTP1 gene polymorphisms with the risk of prostate cancer: a meta-analysis. Cancer Epidemiol Biomarkers Prev 2005; 14: 176-181.

9. Skrętkowicz J, Barańska M, Kaczorowska A, RychlikSych M. Genetic polymorphisms of CYP2D6 oxidation in patients with systemic lupus erythematosus. Arch Med Sci 2011; 7: 864-869.

10. Srivastava DS, Mandhani A, Mittal B, Mittal RD. Genetic polymorphism of glutathione S-transferase genes (GSTM1, GSTT1 and GSTP1) and susceptibility to prostate cancer in Northern India. BJU Int 2005; 95: 170-173.

11. Song DK, Xing DL, Zhang LR, et al. Association of NAT2, GSTM1, GSTT1, CYP2A6, and CYP2A13 gene polymorphisms with susceptibility and clinicopathologic characteristics of bladder cancer in Central China. Cancer Detect Prev 2009; 32 : 416-423.

12. Boccia S, Cadoni G, Sayed-Tabatabaei FA, et al. CYP1A1, CYP2E1, GSTM1, GSTT1, EPHX1 exons 3 and 4, and NAT2 polymorphisms, smoking, consumption of alcohol and fruit and vegetables and risk of head and neck cancer. J Cancer Res Clin Oncol 2008; 134: 93-100.

13. Mo Z, Gao Y, Cao Y, et al. An updating meta-analysis of the GSTM1, GSTT1, and GSTP1 polymorphisms and prostate cancer: a HuGE review. Prostate 2009; 69: 662-688.

14. Vijayalakshmi K, Vettriselvi V, Krishnan M, et al. Polymorphisms at GSTM1 and GSTP1 gene loci and risk of prostate cancer in a South Indian population. Asian Pacific J Cancer Prev 2005; 6: 309-314.

15. Chen B, Cao L, Zhou Y, et al. Glutathione S-transferase T1 (GSTT1) gene polymorphism and gastric cancer susceptibility: a meta-analysis of epidemiologic studies. Dig Dis Sci 2010; 55 : 1831-1838.

16. Longuemaux S, Deloménie C, Gallou C, et al. Candidate genetic modifiers of individual susceptibility to renal cell carcinoma: a study of polymorphic human xenobiotic-metabolizing enzymes. Cancer Res 1999; 59: 2903-2908.

17. Law KM, Brunt EM. The Stomach. In: The Washinton Manual of Surgical Pathology. Humphrey PA, Denner LP, Pfeifer JD (eds.). Wolter Kluwer (Health), Lippincott Williams \& Wilkins, Philadelphia 2012; 206. 
18. Huszno J, Mrochen-Domin I, Zembala-Nożýnska E, et al. Mucin secretion activity of gastric cancer as a prognostic factor: a clinicopathological analysis. Contemp Oncol (Pozn) 2012; 16: 159-164.

19. La Torre G, Boccia S, Ricciardi G. Glutathione S-transferase M1 status and gastric cancer risk: a meta-analysis. Cancer Lett 2005; 217: 53-60.

20. Wang C, Yuan Y, Hunt RH. The association between Helicobacter pylori infection and early gastric cancer: a meta-analysis. Am J Gastroenterol 2007; 102: 1789-1798.

21. Saadat M. Genetic polymorphisms of glutathione S-transferase T1 (GSTT1) and susceptibility to gastric cancer: a meta-analysis. Cancer Sci 2006; 97: 505-509.

22. Boccia S, La Torre G, Gianfagna F, et al. Glutathione S-transferase T1 status and gastric cancer risk: a meta-analysis of the literature. Mutagenesis 2006; 21: 115-123.

23. Alshagga MA, Mohamed N, Nazrun Suhid A, et al. Frequencies of glutathione s-transferase (GSTM1, GSTM3 AND GSTT1) polymorphisms in a Malaysian population. Arch Med Sci 2011; 7: 572-578.

24. Tiwawech D, Chindavijak S, Karalak A, Ishida T. Real-time PCR assay for rapid detection of GSTM1 polymorphism in nasopharyngeal carcinoma patients. Asian Pac J Cancer Prev 2008; 9: 233-238.

25. Chen B, Zhou Y, Yang P, Wu XT. Glutathione S-transferase M1 gene polymorphism and gastric cancer risk: an updated analysis. Arch Med Res 2010; 41: 558-566.

26. Baloglu G, Haholu A, Kucukodaci Z, et al. The effects of tissue fixation alternatives on DNA content: a study on normal colon tissue. Appl Immunohistochem Mol Morphol 2008; 16: 485-492.

27. Tamer L, Ateş NA, Ateş C, et al. Glutathione S-transferase M1, $\mathrm{T} 1$ and $\mathrm{P} 1$ genetic polymorphisms, cigarette smoking and gastric cancer risk. Cell Biochem Funct 2005; 23: 267-272.

\section{Address for correspondence}

Assoc. Prof. Aptullah Haholu MD

Pathology Department

Gulhane Military Medical Academy (GMMA)

Haydarpasa Training Hospital

34668 Uskudar/Istanbul, Turkey

tel. +905326718212

e-mail: ahaholu@gata.edu.tr 